研究

\title{
A Consideration on Grain Growth in WC-Co Fine Grained Hardmetal by Numerical Calculation Based on Alloy Model with Three Kinds of Grain Sizes
}

\author{
Naoki Matsuoka ${ }^{\text {} 1}$, Yoshihiko Doi ${ }^{\text {म } 2}$ and Koji Hayashi ${ }^{\text {म1 }}$ \\ 4 Institute of Industrial Science, the University of Tokyo, 7-22-1 Roppongi, Minato-ku, Tokyo 106-8558. \\ ${ }^{2}$ Tokyo Tungsten Co. Ltd., 5-24-8 Ueno, Taito-ku, Tokyo 110-0005.
}

Received January 16, 1998

\begin{abstract}
SYNOPSIS
In the previous work, we have derived a differential equation for the numerical calculation of the abnormal growth of WC grains in WC-VC-Co fine grained hardmetal, based on grain-dispersed alloy model with two kinds of grain sizes (2-grain-size model) where the rate-determining step of Ostwald ripening of WC grains is the interface reaction, and then numerically estimated the initial sizes of small and large grains where the abnormal grain growth occurs. In this work, first we derived differential equations for calculating each grain size and mean grain size in alloy model with three kinds of grain sizes (3-grain-size model), secondly calculated the large grain size and mean grain size after sintering for $\mathrm{WC}-0.5$ mass $\% \mathrm{VC}-10$ mass\%Co fine grained hardmetal as a function of initial sizes of three kinds of grains and their initial mass fractions, and finally estimated both more detailed conditions for the occurrence or non-occurrence of the abnormal grain growth and the mean grain size.

KEY WORDS

abnormal grain growth, anomalous grain growth, micro-grain, fine grain, hardmetal, cemented carbide, numerical calculation, tungsten carbide, grain growth inhibitor
\end{abstract}

\section{Introduction}

WC-Co base fine grained hardmetals where a small amount of $\mathrm{VC}$ and/or $\mathrm{Cr}_{3} \mathrm{C}_{2}$ are added as inhibitors of the grain growth during sintering are being used as materials for tools such as drills, shear blade and endmill ${ }^{1-3)}$. The mean grain size of fine grained hardmetals on the market are ordinarily set above about $0.5 \mu \mathrm{m}^{1-4)}$, although the hardness and wear resistance tend to increase further with decreasing mean grain size below $0.5 \mu \mathrm{m}$ and the finer WC powders are commercially available. This is because the fracture strength of the alloy considerably drops with decreasing mean grain size below $0.2 \sim 0.3 \mu \mathrm{m}$, due to extremely large $\mathrm{WC}$ grains ${ }^{1)}$ which are generated by abnormal grain growth and act as the fracture source ${ }^{5)}$. The microstructure of such alloy consisting of a large amount of small grains and a considerably small amount of extremely large grains is generally called "abnormal grain microstructure".

The mechanism of the abnormal grain growth or the formation of abnormal grain microstructure is not clear at present, although the following hypotheses for the cause seem to be thought ${ }^{6)}$; (i) wide gain size distribution of WC grains in the raw WC powder or WC+Co green compact, (ii) non-uniform mixing or distribution of the grain growth inhibitor in the compact, (iii) almost intrinsic phenomenon as far as $\mathrm{VC}$ and/or $\mathrm{Cr}_{3} \mathrm{C}_{2}$ are used as the grain growth inhibitor. The clarification of the main cause for the abnormal grain growth is important for the determination of the method for producing fine grained hardmetals with grain size below $0.4 \mu \mathrm{m}$ as well as without abnormal large grains.

Therefore, we have started a numerical simulation study on estimating the mean grain sizes and grain size distribution where the above hypothesis (i) or (iii) becomes the main cause of the abnormal grain growth ${ }^{6}$. As the first step, we have already derived a general equation for the numerical calculation of the growth of large grains based on graindispersed alloy model where two kinds of grains, i.e., a large amount of small grains and a considerably small amount of large grains are dispersed in liquid phase (we call this alloy model "2-grain-size model"), assuming that the ratedetermining step of Ostwald ripening of WC grains is the interface reaction (the precipitation of WC solute in liquid phase onto WC grains) ${ }^{7}$, and numerically estimated the initial small and large grain sizes where the abnormal growth occurs during sintering for WC- 0.5 mass $\%$ VC- 10 mass $\% \mathrm{Co}$ fine grained hardmetal ${ }^{4}$. The calculation results showed that abnormal grain growth substantially occurs when the initial small grain size becomes less than $0.2 \sim 0.1 \mu \mathrm{m}$, 
irrespective of the initial large grain size.

In this study, we used "alloy model with three kinds of grain sizes" or "3-grain-size model" which includes further middle grains in addition to small and large grains in the previous 2-grain-size model ${ }^{6}$. The schematic illustration of this new alloy model is shown in Fig.1. Based on this new model, we first derived general equations for the small, middle and large grain size, $d_{S, t}, d_{M, t}, d_{L, t}$, and mean grain size, $\bar{d}_{t}$, after sintering for time of $t$, assuming that the ratedetermining step of Ostwald ripening is the precipitation as in the previous work ${ }^{6}$. Next, we calculated the values of $d_{L, 3.6}$ and $\bar{d}_{3.6}$ as a function of initial grain sizes $\left(d_{S, 0}, d_{M, 0}\right.$, $\left.d_{L, 0}, \bar{d}_{0}\right)$ and initial grain mass fractions $\left(W_{S, 0}, W_{M, 0}, W_{L, 0}\right)$ or initial number fractions $\left(N_{S, 0}, N_{M, 0}, N_{L, 0}\right)$. Then, we estimated the conditions for the occurrence or non-occurrence of abnormal grain growth in fine grained hardmetals and also for obtaining fine grained hardmetal without abnormal grains and with mean grain size as small as possible.

The importances of the numerical calculation based on this 3-grain-size model are as follows. We can quantitatively estimate by using this model whether the addition or incorporation of middle grains suppresses or accelerates the abnormal grain growth of large grains. Furthermore, this model can simulate the microstructural change in fine grained hardmetal which has more complex grain size distribution than the previous "2-grain-size model", and this will also become a basis for "multi-grainsize model", based on which we will more precisely investigate the above subjects.

\section{Derivation of grain growth equations}

As described later, the numerical calculation showed that small grains disappear in general during sintering in this 3 -grain-size model. Therefore, the grain growth equations before and after the disappearance of small grains were derived separately.

2.1 Equations before disappearance of small grains

As the first step, we derived the growth equations of middle and large grains. Based on the above described assumption that the rate-determining step of Ostwald ripening in this alloy model is the interface reaction as in the previous model ${ }^{6}$, the distribution of concentration $\left(C_{t}\right)$ of solute in liquid phase during sintering at a time of $t$ can be schematically expressed in Fig.2. Namely, the concentration, $C_{t}$, of solute in liquid phase except for at the interfaces between middle or large grains and liquid phase is equal to the largest solubility among solubilities of three kinds of grains ${ }^{6}$, i.e., the solubility of small grain, $C_{S . t}$, which is expressed by the following equation ${ }^{8,9}$.

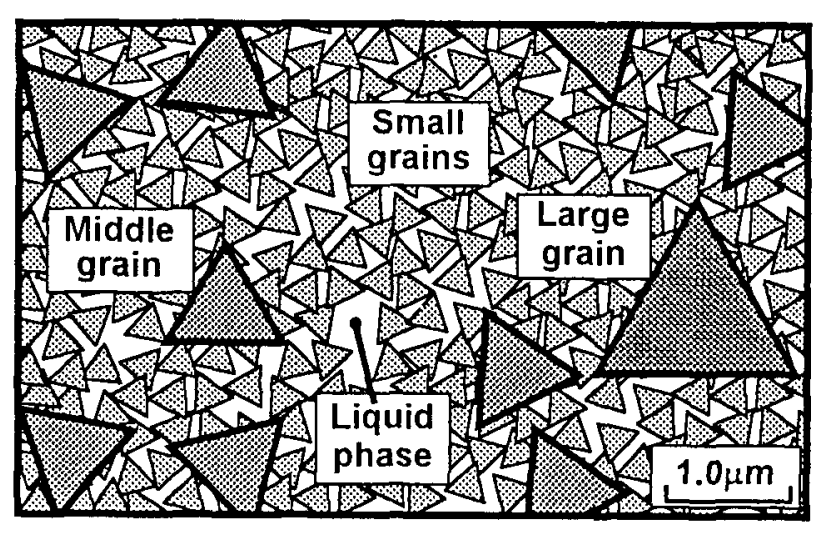

Fig.1 Alloy model with three kinds of grain sizes (or 3-grain-size model) where a large amount of small grains (the equivalent diameter, $\left.d_{s, 0}\right)$, and a small amount of middle grains $\left(d_{M, 0}\right)$ and a considerably small amount of large grains (the diameter, $d_{L, 0}$, is lager than the twice of that of small grains, $d_{S, 0} ; d_{L, d}$ $d_{s, 0} \geqq 2$ ) are dispersed in liquid phase (white matrix).

$$
\begin{aligned}
C_{t} & =C_{S, t} \\
& =C_{0}+2 C_{0}\left(2 \sigma V_{m} / v R T\right)\left(1 / d_{S, t}\right)
\end{aligned}
$$

Here, $C_{0}$ is the equilibrium solubility (molar concentration) of solid phase with flat surface, $\sigma$ is the interface energy between solid and liquid phases, $V_{m}$ is the molar volume of solid phase, $v$ is a correction factor for the deviations of both the solid composition from the stoichiometric value and the grain morphology from sphere ${ }^{10,11)}$ and also for the contiguity of grains, $\mathrm{R}$ is gas constant, and $T$ is the sintering (heating) temperature.

At the interfaces between middle or large grains and liquid phase, their concentrations of the solute in the liquid phase are considered to be their equilibrium solubilities, $C_{M, t}$ and $C_{L, t}$, respectively ${ }^{6)}$, which are given by the followings ${ }^{8,9)}$.

$$
\begin{aligned}
& C_{M_{, t}}=C_{0}+2 C_{0}\left(2 \sigma V_{m} / v R T\right)\left(1 / d_{M_{,},}\right) \\
& C_{L, t}=C_{0}+2 C_{0}\left(2 \sigma V_{m} / v R T\right)\left(1 / d_{L, t}\right)
\end{aligned}
$$

Both middle and large grains grow due to the difference in solubilities between small and middle or large grains when the rate-determining step is the interface reaction ${ }^{8}$. Therefore, the growth differential equations of middle and large grains can be derived in the same way as that of large grains in the previous model ${ }^{6}$.

$$
\begin{aligned}
& d_{M, t+\Delta t}=d_{M, t}+(81 / 32) \cdot K \cdot\left(1 / d_{S, t}-1 / d_{M, t}\right) \cdot \Delta t \\
& d_{L, t+\Delta t}=d_{L, t}+(81 / 32) \cdot K \cdot\left(1 / d_{S, t}-1 / d_{L, t}\right) \cdot \Delta t
\end{aligned}
$$

Here, $K$ is the rate constant in $\bar{d}_{t}^{2}-\bar{d}_{0}{ }^{2}=K$, which is a wellknown equation expressing for the time dependence of mean grain size in the case of the rate-determining step of 


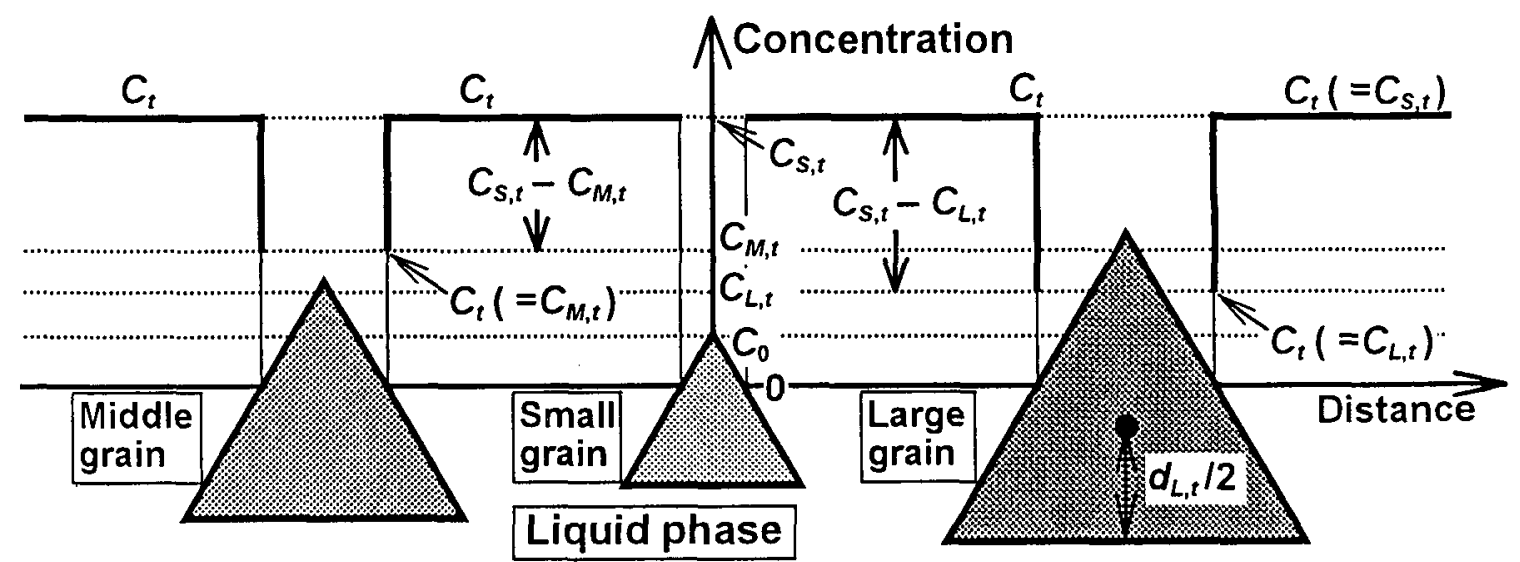

Fig.2 Schematic illustration for distribution of WC solute concentration at a time of $t\left(C_{l}\right)$ in cobalt liquid phase among small, middle and large grains during sintering. The $C_{t}$ in the figure is the one before the disappearance of small grains. After it, $C_{t}$ changes from $C_{S, t}$ to $C_{M, l}$. This is based on the assumption that the rate-determining step of Ostwald ripening is the interface reaction (precipitation). The grain size is defined as the twice of the length of the perpendicular from the center of gravity of the triangle (denoted by " * ) to the base $^{6}$, an example of which is shown for large grain. This model or equations (1) (23) in the text are applicable to general graindispersed alloys having grains with various shapes, although equilateral triangular grains corresponding to WC grain are drawn in the figure.

interface reaction; $K=(32 / 81) 4\left(2 \sigma \kappa C_{0} V_{m}{ }^{2} / v \mathrm{R} T\right)$, where $\kappa$ is interface reaction rate constant ${ }^{8,9)}$.

Next, we derived the decay equation of small grains as follows. Taking into consideration the material balance, i.e., the total volume (or weight) of precipitation from liquid phase onto middle and large grains is equal to the total volume of dissolution from small grains into liquid phase. Then, we first calculated the total volume increment of middle and large grains per unite time, secondly the decay of small grain size. When the weight ratios of small, middle and large grains to the weight of total grains at time of zero, i.e., before sintering, are denoted as $W_{S, 0}, W_{M, 0}$ and $W_{L, 0}\left(W_{S, 0}+W_{M, 0}+W_{L, 0}=1\right)$, respectively, the initial number ratio of each grain, $N_{S, 0}, N_{M, 0}$ and $N_{L, 0}\left(N_{S, 0}+N_{M, 0}+N_{L, 0}=1\right)$, are expressed as follows, respectively.

$$
\begin{aligned}
& N_{S, 0}=\left(6 W_{S, 0} W / \pi \rho d_{S, 0}{ }^{3}\right) / N \\
& N_{M, 0}=\left(6 W_{M, 0} W / \pi \rho d_{M, 0}{ }^{3}\right) / N \\
& N_{L, 0}=\left(6 W_{L, 0} W / \pi \rho d_{L, 0}{ }^{3}\right) / N
\end{aligned}
$$

where $W$ is the total weight of three kinds of grains, $\rho$ is the specific gravity of solid grain and $N$ is the total number of three kinds of grains, which is given by the following.

$$
N=(6 W / \pi \rho)\left(W_{S, 0} / d_{S, 0}{ }^{3}+W_{M, 0} / d_{M, 0}{ }^{3}+W_{L, 0} / d_{L, 0}{ }^{3}\right)
$$

It is needless to say that the number ratio of each grain during sintering, i.e., $N_{S, t}, N_{M, t}$ and $N_{L, t}$, does not change from their initial ratio in contrast with $W_{S, l}, W_{M, t}$ and $W_{L, t}$, because we are now dealing with the case where small grains do not disappear. Therefore, the following relations hold.

$$
\begin{aligned}
& N_{S, t}=N_{S, 0} \\
& N_{M, t}=N_{M, 0} \\
& N_{L, t}=N_{L, 0}
\end{aligned}
$$

The volume of one large grain at times of $t+\Delta t$ and $t$, $V_{L t+\Delta t}$ and $V_{L, t}$, are described by the following equations.

$$
\begin{aligned}
V_{L, t+\Delta t} & =(4 / 3) \pi\left(d_{L t+\Delta \Delta} / 2\right)^{3} \\
& =(\pi / 6) d_{L, t+\Delta t}{ }^{3} \\
V_{L, t} & =(\pi / 6) d_{L, t}{ }^{3}
\end{aligned}
$$

Then, the volume increment, $\Delta V_{L i}$, of one large grain, due to the grain growth during sintering time increment, $\Delta t$, is expressed as the following.

$$
\begin{aligned}
\Delta V_{L, t} & =V_{L, t+\Delta t}-V_{L t} \\
& =(\pi / 6) d_{L, t+\Delta t}{ }^{3}-(\pi / 6) d_{L, t}{ }^{3} \\
& =(\pi / 6) \cdot\left(d_{L, t+\Delta t}{ }^{3}-d_{L, t}{ }^{3}\right)
\end{aligned}
$$

Here, the inside of right bracket in the right side of equation (6) is approximated as follows, because the relation of $d_{L, t+\Delta t}=d_{L, t}+\Delta d_{L, t}$ holds.

$$
\begin{aligned}
d_{L t+\Delta t}{ }^{3}-d_{L, t}{ }^{3} & =\left(d_{L, t}+\Delta d_{L, t}\right)^{3}-d_{L, t}{ }^{3} \\
& =3 d_{L, t}{ }^{2} \cdot \Delta d_{L, t}+3 d_{L, t} \cdot \Delta d_{L, t}{ }^{2}+\Delta d_{L, t}{ }^{3} \\
& \fallingdotseq 3 d_{L, t}{ }^{2} \cdot \Delta d_{L, t}
\end{aligned}
$$

By using the above relation, equation (6) can be approximated as follows.

$$
\Delta \mathrm{V}_{L, t} \doteqdot(\pi / 2) \cdot d_{L, t}{ }^{2} \cdot \Delta d_{L, t}
$$

By rearranging equation (3), the following is obtained. 


$$
\begin{aligned}
\Delta d_{L, t} & =d_{L, t+\Delta t}-d_{L, t} \\
& =(81 / 32) \cdot K \cdot\left(1 / d_{S, t}-1 / d_{L, t}\right) \cdot \Delta t
\end{aligned}
$$

By substituting equation (8) for equation (7), the following equation is derived.

$$
\Delta V_{L, t}=(\pi / 2) \cdot d_{L, t}{ }^{2} \cdot(81 / 32) \cdot K \cdot\left(1 / d_{S, r}-1 / d_{L, t}\right) \cdot \Delta t
$$

Then, the total volume increment of large grains with number of $N_{L, t}\left(=N_{L, 0}\right)$, i.e., $\Delta V_{L, t} \cdot N_{L, t}$, is given by the following equation.

$$
\begin{aligned}
\Delta V_{L, t} \cdot N_{L, t}=(\pi / 2) \cdot(81 / 32) & \cdot K \cdot d_{L, t}{ }^{2} N_{L, t} \\
& \times\left(1 / d_{S, t}-1 / d_{L, t}\right) \cdot \Delta t
\end{aligned}
$$

As for the middle grains, the following equation is obtained in the same way as equation (10).

$$
\begin{aligned}
\Delta V_{M, t} \cdot N_{M, t}=(\pi / 2) \cdot(81 / 32) & \cdot K \cdot d_{M, t}^{2} N_{M, t} \\
& \times\left(1 / d_{S, t}-1 / d_{M, t}\right) \cdot \Delta t
\end{aligned}
$$

Taking into consideration the material balance described above, the following relation holds.

$$
\Delta V_{S, t} \cdot N_{S, t}=-\left(\Delta V_{M, t} \cdot N_{M, t}+\Delta V_{L, t} \cdot N_{L, t}\right)
$$

Where, the mark "-" in the right side means the decrease of the amount. Then,

$$
\Delta V_{S, t}=-\left(\Delta V_{M, t} \cdot N_{M, t}+\Delta V_{L, t} \cdot N_{L, t}\right) / N_{S, t}
$$

The $\Delta V_{S, t}$ can also be approximated as follows by using both the same method for the deviation of equation (7) and the relation of $d_{S, t+\Delta t}=d_{S, t}+\Delta d_{S, t}$.

$$
\begin{aligned}
\Delta V_{S, t} & \fallingdotseq(\pi / 2) \cdot d_{S, t}{ }^{2} \cdot \Delta d_{S, t} \\
& =(\pi / 2) \cdot d_{S, t}{ }^{2}\left(d_{S, t+\Delta t}-d_{S, t}\right)
\end{aligned}
$$

By rearranging this equation, the following is obtained.

$$
d_{S, t+\Delta t}=d_{S, t}+(2 / \pi) \cdot\left(\Delta V_{S, l} / d_{S, t}{ }^{2}\right)
$$

By substituting equations (13), (10) and (11) for equation (15), the following decay differential equation of small grains is obtained.

$$
\begin{aligned}
d_{S, t+\Delta t}= & d_{S, t}-(2 / \pi) \cdot\left(\Delta V_{M, t} \cdot N_{M, t}+\Delta V_{L, t} \cdot N_{L, t}\right) /\left(d_{S, t}{ }^{2} N_{S, t}\right) \\
= & d_{S, t}-(81 / 32) \cdot K \cdot\left(d_{M, t}{ }^{2} N_{M, t} \cdot\left(1 / d_{S, t}-1 / d_{M, t}\right)\right. \\
& \left.+d_{L, t}{ }^{2} N_{L, t} \cdot\left(1 / d_{S, t}-1 d_{L, t}\right)\right\} \cdot \Delta t /\left(d_{S, t}{ }^{2} N_{S, t}\right)
\end{aligned}
$$

where $N_{M, t}$ and $N_{L, t}$ are equal to $N_{M, 0}$ and $N_{L, 0}$, as already shown by equations (4) and (4").

The value of $K$ in equations (2), (3) and (16) is estimated to be $2.18 \times 10^{-2} \mu \mathrm{m}^{2} / \mathrm{ks}\left(7.85 \times 10^{-2} \mu \mathrm{m}^{2} / \mathrm{h}\right)$ in the case of sintering at $1673 \mathrm{~K}$ for WC -0.5 mass $\%$ VC- 10 mass $\%$ Co typical fine grained hardmetal from the experimental data on $\bar{d}_{t}^{2}-t$ relation ${ }^{12)}$, as shown in the previous report ${ }^{6)}$. Then, equations (2), (3) and (16) are rewritten as follows.

$$
\begin{aligned}
d_{M, t+\Delta t}= & d_{M, t}+5.52 \times 10^{-2}\left(1 / d_{S, t}-1 / d_{M, t}\right) \cdot \Delta t \\
d_{L, t+\Delta t}= & d_{L, t}+5.52 \times 10^{-2}\left(1 / d_{S, t}-1 / d_{L, t}\right) \cdot \Delta t \\
d_{S, t+\Delta t}= & d_{S, t}-5.52 \times 10^{-2}\left\{d_{M, t}{ }^{2} N_{M, t} \cdot\left(1 / d_{S, t}-1 / d_{M, t}\right)\right. \\
& \left.+d_{L, t}{ }^{2} N_{L, t} \cdot\left(1 / d_{S, t}-1 / d_{L, t}\right)\right\} \cdot \Delta t /\left(d_{S, t}{ }^{2} N_{S, t}\right)
\end{aligned}
$$

The unit of time, $t$, in these equations is "ks". Furthermore, the arithmetic mean grain size, $\bar{d}_{t}$, is expressed as the following,

$$
\bar{d}_{t}=d_{S, t} \cdot N_{S, t}+d_{M, t} \cdot N_{M, t}+d_{L, t} \cdot N_{L, t}
$$

where $d_{S, t}, d_{M, l}$ and $d_{L, t}$ are calculated by using equations (17) $\sim(19)$, respectively.

2.2 Equations after disappearance of small grains

In the process of computing, the value of $d_{s, 0}$ becomes below zero before the sintering time $(t)$ reaches $3.6 \mathrm{ks}$. This means that small grains disappear during sintering, resulting from the continuous dissolution in the process of Ostwald ripening. After the disappearance, the large grains grow due to the difference in solubility between middle and large grains instead of the difference in solubility between small and large grains, and thus middle grains begin to decay in the opposite way to the growth before the disappearance of small grains. The growth equation of large grains and the decay equation of middle grains can be derived in a similar way to the above equations (3) or (18) and (16) or (19), respectively. The resultant differential equations are as follows.

$$
\begin{aligned}
d_{L, t+\Delta t}= & d_{L, t}+5.52 \times 10^{-2}\left(1 / d_{M, t}-1 / d_{L, t}\right) \cdot \Delta t \\
d_{M, t+\Delta t}= & d_{M, t}-5.52 \times 10^{-2} \\
& \times\left\{d_{L, t}{ }^{2} N_{L, t} \cdot\left(1 / d_{M, t}-1 / d_{L, t}\right)\right\} \cdot \Delta t /\left(d_{M, t}{ }^{2} N_{M, t}\right)
\end{aligned}
$$

where $N_{L, t}$ and $N_{M, t}$ is given by using equations similar to equations (4), (4') and (4"). And the mean grain size, $\bar{d}_{t}$, is expressed by the following.

$$
\bar{d}_{i}=d_{M, r} \cdot N_{M, t}+d_{L, t} \cdot N_{L, t}
$$

It is needless to say that all these equations, i.e., equations (1) (23), are applicable to the grain growth of general grain-dispersed alloys having the rate-determining step of interface reaction as well as WC-Co fine grained hardmetal, by changing $K$ value or $5.52 \times 10^{-2}$ to the value appropriate to each alloy.

\section{Results of numerical calculation and consideration}

The computer program (The language is Microsoft Quick Basic. The number of lines is about 400 ) for the numerical calculation of both each grain size and the mean grain size 


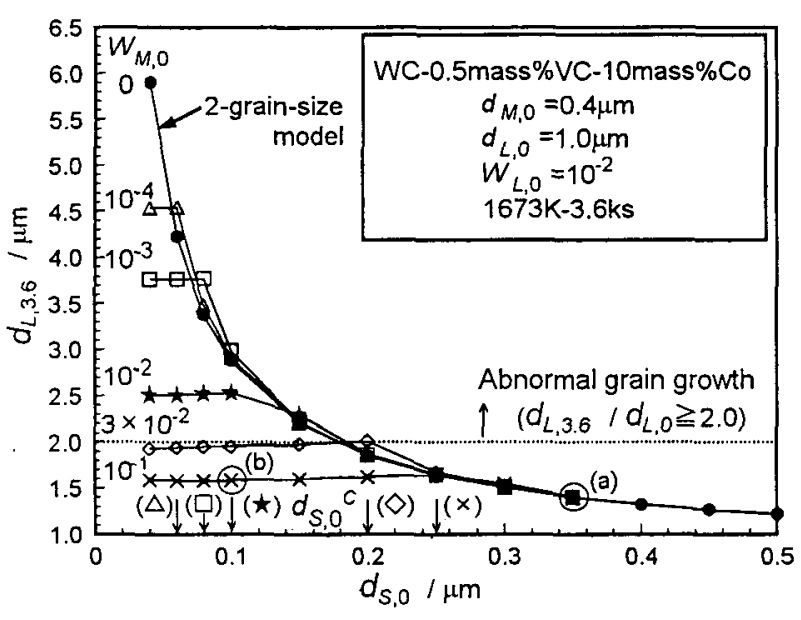

Fig.3 Effect of $W_{M, 0}$ on relation between $d_{L, 3.6}$ and $d_{S, 0}$, which was obtained by numerical calculation with equations (18) and (21). The $\bar{d}_{0}$ is almost equal to $d_{s, 0}$ for all cases in this study, mainly due to a large amount of small grains. The data points do not exist above $0.4 \mu \mathrm{m}$ in 3 -grain-size model, because $d_{s, 0}$ should be smaller than $d_{M, 0}(0.4 \mu \mathrm{m})$ from the definition of $d_{S, 0}<d_{M, 0}(0.4 \mu \mathrm{m})$. The sintering time dependence of each grain size at the conditions of circled points (a) and (b) in the case of $W_{M, 0}=10^{-1}$ are shown in next figures, i.e., Fig. 4 (a) and (b).

after sintering for $t+\Delta t$ by using equations (17) (23) was made, taking into consideration the disappearance of small grains during sintering and then installed into a computer (NEC Xa 16). Then, the values of $d_{S, t}, d_{M, t}, d_{L, t}$ and $\bar{d}_{t}$ for WC-0.5mass\%VC-10mass\%Co fine grained hardmetal were calculated in the case of the usual sintering condition, i.e., at $1673 \mathrm{~K}$ for $3.6 \mathrm{ks}$, as a function of $d_{s, 0}$ (The range is $0.04 \sim 0.5 \mu \mathrm{m}$; the step is 0.02 or $0.1 \mu \mathrm{m}$ ), $d_{M, 0}$ (The range is $0.4 \sim 0.8 \mu \mathrm{m}$; the step is $0.1 \mu \mathrm{m}$ ) and $W_{M .0}$ (The range is in

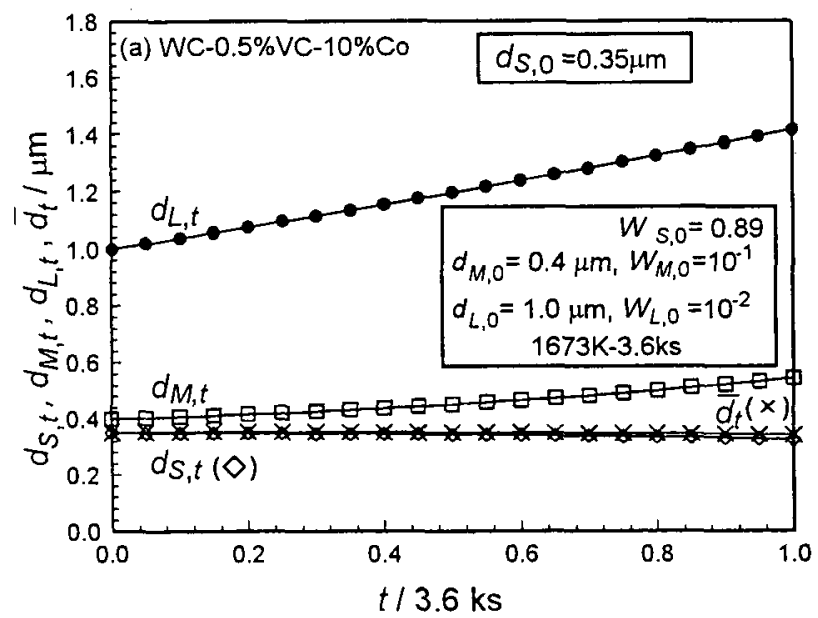

general $10^{-4} \sim 10^{-1}$, and the range of the number ratio which varies with $d_{M, 0}$ even at a definite $W_{M, 0}$ is $2 \times 10^{-7} \sim 2 \times 10^{-3}$ ). The $d_{L, 0}$ and $W_{L, 0}$ were set in general to be $1.0 \mu \mathrm{m}$ and $10^{-2}$ (The range of the number ratio is $7.2 \times 10^{-7} \sim 1.4 \times 10^{-3}$ ), respectively. Under these conditions, the initial mean grain size, $\bar{d}_{0}$, always nearly equal to $d_{s, 0}$. The increment of $t, \Delta t$, was set to be $3.6 \times 10^{-3} \mathrm{ks}$.

Fig. 3 shows the effect of $W_{M, 0}$ on the relation between $d_{L, 3.6}$ (large grain size after sintering) and $d_{s, 0}$ under the condition of $\left(d_{M, 0}, d_{L, 0}, W_{L, 0}\right)=\left(0.4 \mu \mathrm{m}, 1.0 \mu \mathrm{m}, 10^{-2}\right)$. The previous result based on 2-grain-size model ${ }^{6}$, which corresponds to the case of $W_{M, 0}=0$ in 3-grain-size model, is also shown in the figure. It is noted that $d_{L, 3.6}$ becomes nearly constant for each $W_{M, 0}$ below a critical value $(0.06$ $\sim 0.25 \mu \mathrm{m}$ ) of $d_{s, 0}$, which was denoted as $d_{S, 0}{ }^{C}$; a horizontal line appears for each $W_{M, 0}$. This is in good contrast with the result that $d_{L, 3.6}$ in 2-grain-size model always increases with decreasing $d_{S, 0}$, irrespective of the value of $d_{S, 0}$. With increasing $W_{M, 0}$, the $d_{L, 3,6}$ of the horizontal line, i.e., $d_{L, 3.6}$ below $d_{s, 0}{ }^{c}$ becomes smaller and the value of $d_{s, 0}{ }^{c}$ increases. Furthermore, the results based on this 3-grain-size model approach to the result based on 2-grain-size model with decreasing $W_{M, 0}$, which manifests the coherency between two kinds of grain-size models.

The cause for the appearance of the horizontal line or $d_{s, 0}{ }^{c}$ is the disappearance of small grains as follows. Fig.4 (a) and (b) show the dependence of $d_{S, t}, d_{M, t}, d_{L, t}$ and $\bar{d}_{t}$ on sintering time $(t)$ under the conditions of circled points (a) and (b) in the Fig. 3 where the $d_{s, 0}$ are $0.35 \mu \mathrm{m}$ and $0.1 \mu \mathrm{m}$, respectively. Fig.4 (a) shows that $d_{S, t}$ does not become zero, i.e., the small grains do not disappear during sintering, when

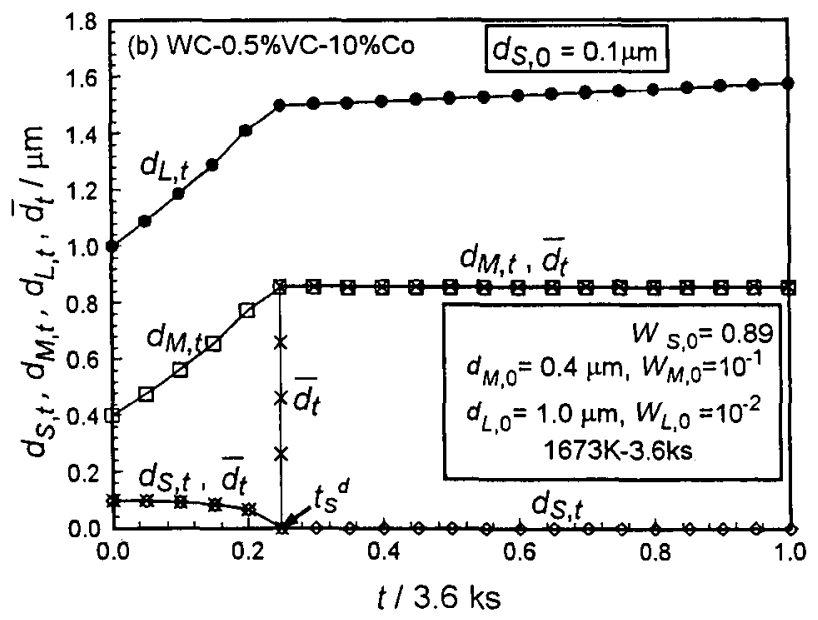

Fig.4 (a), (b) Effect of $d_{s, 0}$ on time dependence of $d_{S,}, d_{M, l}, d_{L,}$, and $\bar{d}_{i}$, which were obtained by numerical calculation with equations (17) $\sim$ (20) before the disappearance of small grains and with equations (21) $\sim(23)$ after it. The number fractions of small, middle and large grains $\left(N_{S, 0}, N_{M, 0}\right.$ and $\left.N_{L, 0}\right)$ corresponding to $W_{S, 0}, W_{M, 0}$ and $W_{L, 0}$ shown in the figures (a) and (b) are $\left(9.2958 \times 10^{-1}, 6.997 \times 10^{-2}\right.$ and $\left.4.5 \times 10^{-4}\right)$ and $\left(9.9823 \times 10^{-1}, 1.75 \times 10^{-3}\right.$ and $\left.1.1 \times 10^{-5}\right)$, respectively. 
the $d_{S, 0}$ is $0.35 \mu \mathrm{m}$. The $d_{M, t}$ and $d_{L, t}$ are both continuously increase with increasing $t$. On the other hand, when the $d_{s, 0}$ is $0.1 \mu \mathrm{m}$, the $d_{S, t}$ becomes zero during sintering as shown in Fig.4 (b); small grains disappear at about $0.25 \times 3.6 \mathrm{ks}$. Such disappearance of small grains during sintering were observed in the range of $d_{s, 0}$ below $0.25 \mu \mathrm{m}$. On the other hand, the $d_{M, t}$ and $d_{L, t}$ relatively sharply increase before the disappearance time of small grains, $t_{s}{ }^{d}$, with increasing $t$, but hardly vary after $t_{s}{ }^{d}$. This shows that the contribution of small grains to the growth of middle and large grains is large and that the contribution of middle grains to the growth of large grains is considerably small. And $\bar{d}_{t}$ is nearly equal to $d_{S . t}$ before $t_{s}{ }^{d}$, abruptly increases at $t_{s}{ }^{d}$, becomes nearly equal to $d_{M, t}$ and does not increase but slightly decreases after $t_{s}{ }^{d}$. The computation also showed that $t_{s}{ }^{d}$ decreases with increasing $W_{M, 0}$ as well as with decreasing $d_{s, 0}$. These results clearly indicate that the cause for the appearance of the horizontal line or $d_{S, 0}{ }^{c}$ is the disappearance of small grains during sintering, i.e., before $1.0 \times 3.6 \mathrm{ks}$. It is also clear that the above mentioned decrease of $t_{s}{ }^{d}$ with increasing $W_{M, 0}$ corresponds to the decrease of the contribution of small grains to the growth of large grains, as also suggested by equations (3) (18). Namely, the rate of the decay of small grains increases with increasing $W_{M, 0}$ $\left(N_{M, 0}, N_{M, t}\right)$ as indicated by equations (16) or (19) and thus $t_{s}{ }^{d}$ or the contribution of small grains becomes smaller. This is the cause for the above mentioned results on the variations of value of $d_{L, 3.6}$ below $d_{S, 0}{ }^{C}$ and the value of $d_{S, 0}{ }^{C}$ in Fig,3.

The results that both $d_{M, t}$ and $d_{L, t}$ relatively sharply increase with increasing $t$ before $t_{s}{ }^{d}$ are of course due to the large driving force caused by the existence of small grains. The results that both $d_{M, t}$ and $d_{L, t}$ hardly vary after $t_{S}{ }^{d}$ are due to the phenomenon that the growth of large grains is driven by the small difference in solubility between middle and large grains after the disappearance of small grains. The results that $\bar{d}_{\mathrm{q}}$ gradually decreases with increasing $t$ before $t_{s}{ }^{d}$, then abruptly increases at $t_{s}{ }^{d}$, and slightly decreases are due to the phenomenon that the value of $\bar{d}_{t}$ is mainly controlled by the size $\left(d_{S, t}\right)$ of small grains with the largest weight or number ratio before $t_{s}{ }^{d}$ and mainly by the size $\left(d_{M, t}\right)$ of middle grains after $t_{s}{ }^{d}$.

"The abnormal grain growth" of the large grains was defined in this study as the growth satisfying the following condition, as in the previous model ${ }^{6}$,

$$
d_{L, 3.6} / d_{L, 0} \geqq 2
$$

Then, it can be said that the abnormal grain growth in this case is the one where $d_{L, 3.6}$ becomes larger than $2.0 \mu \mathrm{m}$, because $d_{L, 0}$ is set to be $1.0 \mu \mathrm{m}$. In Fig.3, the critical value of $d_{L, 3,6}=2.0 \mu \mathrm{m}$ is drawn with a horizontal dotted line. It is noted that the $d_{L, 3.6}$ below $d_{S, 0}{ }^{c}$ decreases with increasing $W_{M, 0}$ and becomes less than $2.0 \mu \mathrm{m}$ in the case where $W_{M, 0}$ exceeds about $3 \times 10^{-2}$ irrespective of $d_{s, 0}$; the abnormal grain growth does not occur at these values of $W_{M, 0}$ and $W_{L, 0}$, although it occurs at $d_{s, 0}$ less than about $0.1 \sim 0.2 \mu \mathrm{m}$ in 2-grain-size model. Therefore, it can be said that the incorporation of middle grains to 2-grain-size model suppresses the occurrence of the abnormal grain growth at $d_{s, 0}$ below about $0.1 \sim 0.2 \mu \mathrm{m}$.

Fig. 5 shows the effect of $W_{M, 0}$ on the relation between $\bar{d}_{3.6}$ (mean grain size after sintering) and $d_{S, 0}$ under the same condition as in Fig.3. In 2-grain-size model $\left(W_{M, 0}=0\right), \bar{d}_{3: 6}$ always decreases with decreasing $d_{S, 0}$ due to the considerably small amount of large grains, although the abnormal grain growth of large grains occurs below 0.2 $0.1 \mu \mathrm{m}$ as mentioned above. On the other hand, $\bar{d}_{3.6}$ in 3 grain-size model decreases with decreasing $d_{s, 0}$ above a certain critical value of $d_{S, 0}$ (which is equal to the above $d_{s, 0}{ }^{C}$ ), but suddenly increases at $d_{S, 0}{ }^{C}$ and becomes nearly constant below $d_{S, 0}{ }^{C}$. It is needless to say that such results at and below $d_{s, 0}{ }^{c}$ are caused by the disappearance of small grains; the main determining factor of $\bar{d}_{t}$ changes from $d_{S, t}$ to $d_{M, t}$ at $d_{S, 0}{ }^{C}$, as above described in relation to Fig.4 (b). The $\bar{d}_{3.6}$ below $d_{S, 0}{ }^{C}$ decreases with increasing $W_{M, 0}$ and becomes $1.3 \mu \mathrm{m}$ at $W_{M, 0} \leqq 3 \times 10^{-2}$ where abnormal grain growth becomes not to occur as described above. It is noted, however, that such mean grain size cannot be called fine grain size.

The above decrease of $\bar{d}_{3.6}$ with increasing $W_{M, 0}$ seems at

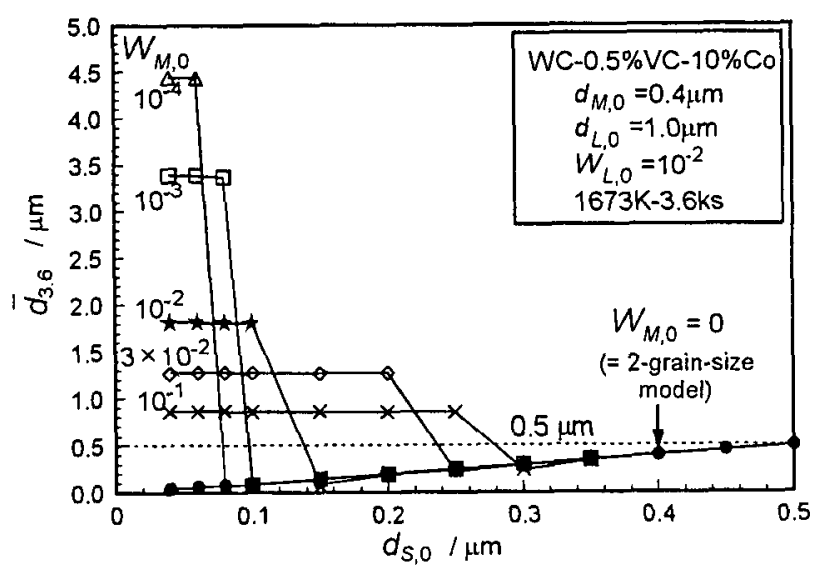

Fig.5 Effect of $W_{M, 0}$ on relation between $\bar{d}_{3.6}$ (mean grain size after sintering at $1673 \mathrm{~K}$ for $3.6 \mathrm{ks}$ ) and $d_{s, 0}$ in the same case as in Fig.3, obtained by numerical calculation with equations (20) and (23). The dotted line at $\bar{d}_{3,6}=0.5 \mu \mathrm{m}$ shows the minimum mean grain size of usual commercial fine grained hardmetals. The position of $d_{s, 0}{ }^{c}$, which are the same as in Fig.3, are not shown in this figure. 
first sight to be peculiar, when we only think that the $\bar{d}_{3.6}$ depends on $W_{M, 0}$, i.e., the amount of middle grains having the grain size larger than small grain. This, however, becomes to be understandable, when we also think the following two things; (1) $\bar{d}_{3.6}$ is mainly determined by $d_{M, 3.6}$ after the disappearance of small grains, and (2)the contribution of small grains to the increase of middle grain size (i.e., $d_{M, 3.6}$ or $\bar{d}_{3.6}$ ), in other words, $t_{s}{ }^{d}$ becomes smaller with increasing $W_{M, 0}$, because $N_{M, 0}$ or $N_{M, t}$ increases and thus the amount of precipitation of the solute fed from small grains onto each middle grain becomes smaller in the case of disappearance of small grains with increasing $W_{M, 0}$.

Fig. 6 shows the effect of $d_{M, 0}$ on the relation between $d_{L, 3.6}$ (large grain size after sintering) and $d_{S, 0}$, where $W_{M, 0}$ is $10^{-1}$ (10mass\%. The number ratio is $2 \times 10^{-7} \sim 2 \times 10^{-3}$ in the case of $d_{S, 0}=0.2 \mu \mathrm{m}$ ). The $d_{L, 3.6}$ increases up to $2.0 \mu \mathrm{m}$ with increasing $d_{M, 0}$ from the above $0.4 \mu \mathrm{m}$ to $0.8 \mu \mathrm{m}$ under the indicated conditions. Fig.7 shows the effect of $d_{M, 0}$ on the relation between $\bar{d}_{3.6}$ (mean grain size after sintering) and $d_{s, 0}$ under the same condition as in Fig.6. The $\bar{d}_{3.6}$ below $d_{S, 0}{ }^{C}$ increases with increasing $d_{M, 0}$ from the above $0.4 \mu \mathrm{m}$ to $0.8 \mu \mathrm{m}$. These results indicate that the increasing of $d_{M, 0}$ has a negative effect for decreasing mean grain size as well as large grain size. These variations of $d_{L, 3.6}$ and $\bar{d}_{3.6}$ with increasing $d_{M, 0}$ seems at first sight to be also peculiar when we only think that the difference in equilibrium solubility between large and middle grains which is the driving force for the growth of large grains after the disappearance of small grains decreases with increasing $d_{M, 0}$. This, however, also becomes to be understandable by considering the followings; (1) The $d_{L, 3.6}$ and $\bar{d}_{3.6}$ are mainly determined by the growth amount of large and middle grains, respectively, which are mainly controlled by the contribution of small

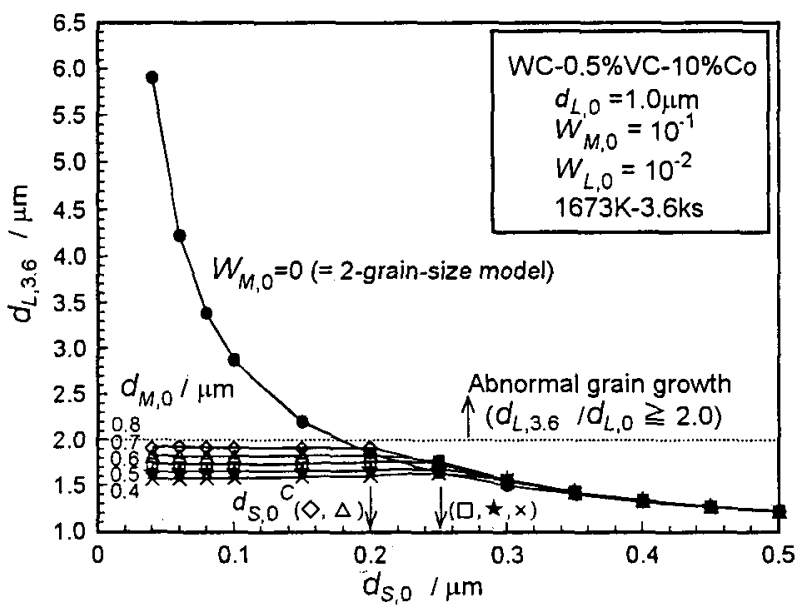

Fig.6 Effect of $d_{M, 0}$ on relation between $d_{L, 3.6}$ and $d_{S, 0}$, obtained by numerical calculation with equations (18) and (21). grains, (2) Such contributions of small grains increase with increasing $t_{s}{ }^{d}$, (3) The $t_{s}{ }^{d}$ becomes longer with increasing $d_{M, 0}$ because $N_{M, 0}$ becomes smaller with increasing $d_{M, 0}$ at a definite $W_{M, 0}$ as shown by equation (4) and thus the dissolution rate of small grains becomes smaller.

Fig. 8 shows the schematic illustrations of microstructures of WC-0.5mass\%VC-10mass\%Co fine grained hardmetal after sintering as a function of $W_{M, 0}$, and $d_{s, 0}$ under the condition of $\left(d_{M, 0}, d_{L, 0}, W_{L, 0}\right)=\left(0.4 \mu \mathrm{m}, 1.0 \mu \mathrm{m}, 10^{-2}\right)$. In the figure, the ratio of the large grain size to the matrix grain size, $d_{L, 3.6} d d_{\text {marix }, 3.6}$, and $\bar{d}_{3.6}$ are shown. Here, the matrix grain is defined to be one which is the smallest and the most numerous. Thus, the matrix grain in this case is small grain before the disappearance of small grain, but middle grain after it. Therefore, the microstructure should be evaluated with $\bar{d}_{3.6}$ and $d_{L, 3.6} / d_{\text {marix } 3.3 .6}$ in 3-grain-size model, instead of $\bar{d}_{3.6}$ and $d_{L, 3.6} / d_{S, 3.6}$ in the previous 2-grain-size model ${ }^{6}$. Namely, in 2-grain-size model, abnormal grain microstructure which consists of a large amount of small grains and a considerably small amount of extremely large grains was defined as microstructure where $d_{L, 3.6} / d_{S, 3.6}$ is larger than 4.0. Therefore, the cause for abnormal grain microstructure in 2-grain-size model is the abnormal grain growth of large grain. However, the abnormal grain growth of large or middle grains in 3-grain-size model does not necessarily cause the abnormal grain microstructure, because the small grains disappear in some cases during sintering, an example of which can be seen in the case of $\left(d_{s, 0}, W_{M, 0}\right)=\left(0.1 \mu \mathrm{m}, 10^{-1}\right)$; the microstructure is not abnormal grain microstructure, although middle or large grains abnormally grow.

Therefore, the definition of abnormal grain microstructure in 3-grain-size model should be changed to be as the microstructure where $d_{L, 3.6} / d_{\text {marrix }, 3.6}$ is larger than 4.0. The judgment based on this definition for the occurrence and non-occurrence of abnormal grain growth

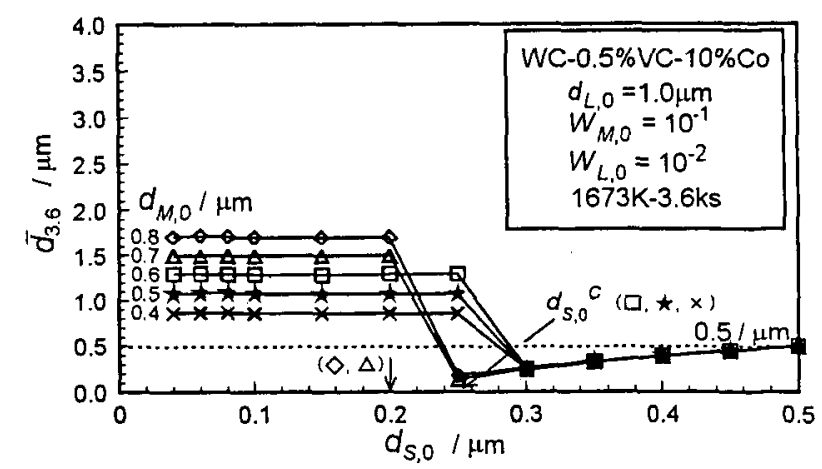

Fig.7 Effect of $d_{M, 0}$ on relation between $\bar{d}_{3.6}$ and $d_{s, 0}$, obtained by numerical calculation with equations (20) and (23). 


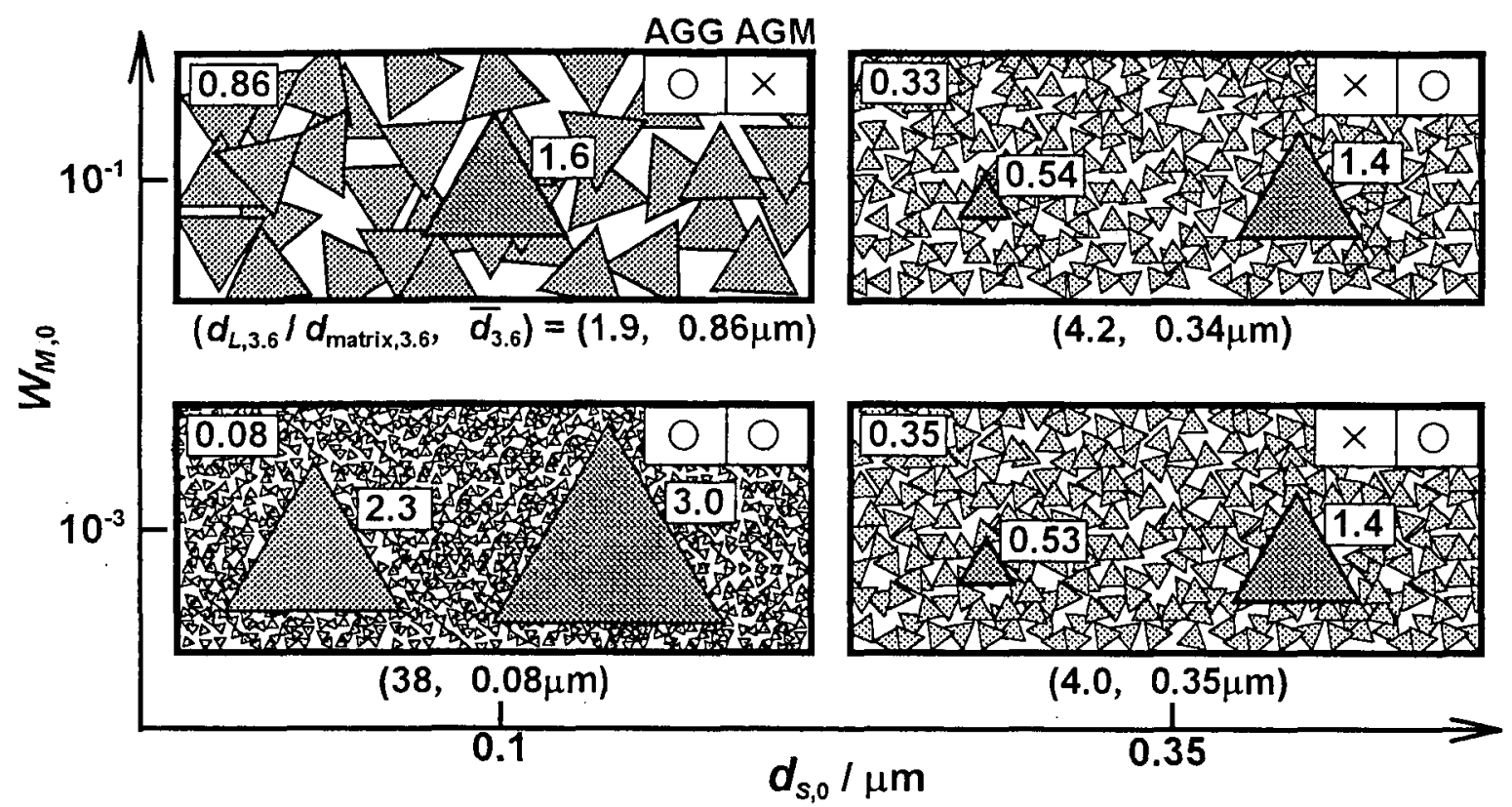

Fig.8 Schematic illustrations of microstructures of WC-0.5mass \%VC-10mass \%Co fine grained hardmetal after sintering at $1673 \mathrm{~K}$ for $3.6 \mathrm{ks}$ as a function of $d_{S, 0}$ and $W_{M, 0}$ under the condition of $\left(d_{M, 0}, d_{L, 0}, W_{M, 0}\right)=\left(0.4 \mu \mathrm{m}, 1.0 \mu \mathrm{m}, 10^{-2}\right)$, which are made from data in Figs.3, 5, 6 and 7. Numerals in rectangles inside the microstructures indicate each grain size $[\mu \mathrm{m}]$. The marks " $\bigcirc$ " and " $\times$ " indicate the occurrence and non-occurrence of abnormal grain growth (AGG, $d_{L, 3,6} / d_{L, 0}$ or $d_{M, 3.6} / d_{M, 0} \geqq 2.0$ ) or abnormal grain microstructure (AGM, $d_{L, 3.6} /$ $d_{\text {matix }, 3.6}$ or $d_{M, 3.6} / d_{\text {matrix }, 3.6} \geqq 4.0$ ), respectively.

(AGG) or abnormal grain microstructure (AGM) are denoted by " $\bigcirc$ " and " $\$ " respectively in Fig.8. Thus, we can say the followings; (1) The incorporation of middle grains above about $3 \times 10^{-2}$ (3mass\%) effectively suppress the abnormal grain growth of large grains, as shown in the case of $\left(d_{S, 0}, W_{M, 0}\right)=\left(0.1 \mu \mathrm{m}, 10^{-1}\right)$ where $d_{L, 3.6} / d_{L, 0}=1.6$. (2) The microstructure in the case of $\left(d_{S, 0}, W_{M, 0}\right)=\left(0.1 \mu \mathrm{m}, 10^{-1}\right)$ cannot be said abnormal grain microstructure $\left(d_{L, 3.6}\right)$ $d_{\text {matrix }, 3.6}=1.9$ ), although middle grains abnormally grow $\left(d_{M, 3 . \delta} d d_{M, 0}=2.2\right)$. In addition, the microstructure in the cases of $\left(d_{S, 0}, W_{M, 0}\right)=\left(0.35 \mu \mathrm{m}, 10^{-3}\right)$ and $\left(0.35 \mu \mathrm{m}, 10^{-1}\right)$ are abnormal grain microstructures $\left\{\left(d_{L, 3.6} / d_{\text {matrix,3.6. }}\right.\right.$ and $\left.d_{M, 3.6}\right\}$ $\left.d_{\text {matrix,3.6 }}\right)=(4.0,1.5)$ and $\left.(4.3,1.6)\right\}$, which, however, are both not due to the abnormal grain growth $\left(d_{L, 3.6} / d_{L, 0}\right.$ are both 1.4), but mainly due to the existence of large grains in the raw powder $\left(d_{L, 0} d d_{\text {matrix }, 0}\right.$ are both 2.9$)$. In short, it is shown that the incorporation of an appropriate amount of middle grains suppresses the abnormal grain growth of large grains below $d_{s, 0}=0.1 \sim 0.2 \mu \mathrm{m}$, but increases the mean grain size up to the value which cannot be called fine grain size.

Therefore, it is suggested that WC-0.5mass\%VC10 mass $\%$ Co fine grained hardmetal having the mean grain size below $0.1 \sim 0.2 \mu \mathrm{m}$ without abnormal grains is hardly produced, only by controlling the grain size distribution of the raw WC powder. In other words, it was suggested that the mean grain size in the raw WC powder or in the green compact of the alloy have to or is enough to be above about $0.3 \mu \mathrm{m}$ for the stable production of WC $-0.5 \mathrm{mass} \% \mathrm{VC}-$ 10 mass $\%$ Co fine grained hardmetal. The present state that the minimum value of mean grain size of commercial fine grained hardmetals is $0.5 \mu \mathrm{m}$ seems to be reasonable. We think that the finding of new grain growth inhibitors having the $K$ of less than $0.336 \times 10^{-2} \mu \mathrm{m}^{2} / \mathrm{ks}^{6}$ is necessary for fabricating finer grained hardmetals without abnormal grain microstructure, as suggested in the previous study ${ }^{6}$. The details, however, should be considered on the basis of multigrain-size model, which corresponds to the actual commercial raw WC powder. We will start this study, based on 2- and 3-grain-size models.

\section{Conclusion}

The differential equations for numerical calculation of each grain sizes $\left(d_{S, t}, d_{M, t}, d_{L, t}\right)$ during sintering in graindispersed alloy model with three kinds of initial grain sizes $\left(d_{S, 0}, d_{M, 0}, d_{L, 0}\right)$, which include " $K$ " in well-known equation of $\bar{d}_{t}^{2}-\bar{d}_{0}^{2}=K t$, were derived for the case where the ratedetermining step of grain growth is the interface reaction or precipitation in the same way as in previous 2-grainsize model. Then, the large grain size $\left(d_{L, 3.6}\right)$ and mean grain size $\left(\bar{d}_{3.6} ;\right)$ of WC-0.5mass\%VC-10mass\%Co fine grained hardmetal sintered at $1673 \mathrm{~K}$ for $3.6 \mathrm{ks}$ were calculated by using the equations as a function of $\left\{d_{S, 0}, d_{M, 0}\right.$, 
$\bar{d}_{0}$ (initial mean grain size; $\left.\left.\fallingdotseq d_{S, 0}\right)\right\}$ and initial grain weight fractions $\left(W_{s, 0}, W_{M, 0}\right)$, or initial number fractions $\left(N_{S, 0}, N_{M, 0}\right)$ at the definite values of $\left(d_{L, 0}, W_{L, 0}\right)=\left(1.0 \mu \mathrm{m}, 10^{-2}\right)$, in order to estimate the conditions for obtaining fine grained hardmetal without abnormal grains and with mean grain size as small as possible.

(1) Derivation of numerical calculation equations

(1-1) Equations before disappearance of small grains; $d_{M, t+\Delta t}=d_{M, t}+(81 / 32) K\left(1 / d_{S, r}-1 / d_{M, t}\right) \Delta t ; d_{L, t+\Delta t}=d_{L, t}+(81 / 32) K(1 /$ $\left.d_{S, r}-1 / d_{L, t}\right) \Delta t ; d_{S, t+\Delta}=d_{S, t}-(81 / 32) K\left\{d_{M, t}{ }^{2} N_{M, t}\left(1 / d_{S, t}-1 / d_{M, t}\right)+d_{L, t}{ }^{2}\right.$ $\left.\times N_{L, t}\left(1 / d_{S, t}-1 / d_{L, t}\right)\right\} \Delta t /\left(d_{S, t}{ }^{2} N_{S, t}\right) ; \bar{d}_{t}=d_{S, t} N_{S, t}+d_{M, t} N_{M, t}+d_{L, t} N_{L, t}$

(1-2) Equations after disappearance of small grains; $d_{L, t+\Delta t}=d_{L, t}+(81 / 32) K\left(1 / d_{M, t}-1 / d_{L, t}\right) \Delta t ; d_{M, t+\Delta t} d_{M, t}-(81 /$ 32) $K\left\{d_{L, t}{ }^{2} N_{L, t}\left(1 / d_{M, r}-1 / d_{L, t}\right)\right\} \Delta t /\left(d_{M, t}{ }^{2} N_{M, t}\right) ; \bar{d}_{i}=d_{M, t} N_{M, t}+d_{L, t} N_{L, t}$ The value of $K$ in these equations is $2.18 \times 10^{-2} \mu \mathrm{m}^{2} / \mathrm{ks}$ in the case of sintering at $1673 \mathrm{~K}$ for $\mathrm{WC}-0.5 \mathrm{mass} \% \mathrm{VC}-$ 10 mass $\%$ Co fine grained hardmetal.

(2) Results of numerical calculation

(2-1) The $d_{L, 3,6}$ increases with decreasing $d_{S, 0}$ in the same way as in 2-grain-size model above a certain critical value, $d_{S, 0}{ }^{C}$, but becomes nearly constant below $d_{s, 0}{ }^{C}$, irrespective of $W_{M, 0}$ and $d_{M, 0}$. This is because the small grains disappear during sintering below $d_{s, 0}{ }^{C}$ and the contribution of middle grains to the growth of large grains is considerably small, compared with that of small grains. The value of $d_{L, 3.6}$ decreases with increasing $W_{M, 0}$ and with decreasing $d_{M, 0}$. (2-2) The $\bar{d}_{3.6}$ decreases with decreasing $d_{s, 0}$ above $d_{s, 0}{ }^{c}$, but suddenly becomes larger at $d_{S, 0}{ }^{C}$ and nearly constant below $d_{S, 0}{ }^{C}$, irrespective of $W_{M, 0}$ and $d_{M, 0}$. This is in good contrast with the result in 2-grain-size model that $\bar{d}_{3.6}$ always decreases with decreasing $d_{S, 0}$, due to a considerably small amount of large grains. The cause is the same as the above. The $\bar{d}_{3.6}$ below $d_{S, 0}{ }^{C}$ decreases with increasing $W_{M, 0}$ and with decreasing $d_{M, 0}$.

(2-3) The numerical calculation suggested that the incorporation of an appropriate amount of middle grains to the mixed powder with a large amount of small grains and a considerably small amount of large grains suppresses the abnormal grain growth of large grains or the formation of abnormal grain microstructure at $d_{s, 0}$ below $0.1 \sim 0.2 \mu \mathrm{m}$, but increases the mean grain size up to the value which cannot be called fine grain size. Namely, it was suggested that the mean grain size of WC raw powders should be above about $0.3 \mu \mathrm{m}$ for the stable production of WC$0.5 \mathrm{mass} \% \mathrm{VC}-10 \mathrm{mass} \% \mathrm{Co}$ fine grained hardmetal.

\section{References}

1) H.Suzuki: "Hardmetals and Sintered Hard Materials Fundamentals and Applications-", Edited by H.Suzuki, Maruzen Co., Tokyo, (1986)249-256.

2) G.E.Spriggs: "A History of Fine Grained Hardmetal", Int. J. of Refractory Metals and Hard Materials, 13(1995)241-255.

3) Z.Fang and J.W.Eason: "Study of Nanostructured WCCo Composites", Int. J. of Refractory Metals and Hard Materials, 13(1995)297-303.

4) K.Hayashi, Y.Fuke and H.Suzuki: "Effects of Addition Carbides on the Grain Size of WC-Co Alloy", J. Japan Inst. Powder and Powder Metallurgy", 19(1972)67-71.

5) H.Suzuki and K.Hayashi: "Strength of WC-Co Cemented Carbides in Relation to their Fracture Sources", J. Japan Inst. Metals, 38(1974)1013-1019; Planseeberichte Pulvermet., 23(1975)24-36.

6) N.Matsuoka and K.Hayashi: "A Consideration on Abnormal Grain Growth in Fine Grained WC-Co Hardmetal by Numerical Calculation-Based on Alloy Model with Two Kinds of Grain Sizes-", Proceedings of $14^{\text {th }}$ International Plansee Seminar '97 vol 2., G.Kneringer, et al., Reutte, Austria, 1997, Plansee AG., RWF, Werbegesellschaft m.b.H., (1997)609-621.

7) K.Hayashi: "Handbooks of Ultrafine Powder", Fuji Technosystem, Tokyo, (1990)509-516.

8) C.Wagner: "Theorie der Alterung von Niederschlägen durch Umlösen", Z.Electrochem., 65(1961)581-591.

9) R.M.German: "Liquid Phase Sintering", Plenum Press, New York and London, (1985)138.

10) C.A.Johnson: "Generalization of the Gibbs-Thomson Equation", Surface Science, 3(1965)429-444.

11) A.J.Ardell: "The Effect of Volume Fraction on Particle Coarsening; Theoretical Considerations", Acta. Met., 20(1972)61-71.

12) H.Suzuki, Y.Fuke and K.Hayashi: "Grain Size of WC in Sintered WC-VC-Co Alloys", J. Japan Inst. Powder and Powder Metallurgy, 19(1972)106-113. 